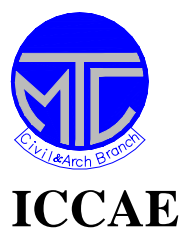

Military Technical College

Kobry Elkobbah, Cairo, Egypt

\author{
6hㅡ International Conference \\ On Civil \& Architecture \\ Engineering
}

\title{
Impact of Sulfate Ion on Dolomite Concrete Performance
}

\author{
W. M. Zadeldin* \\ S. A. Mazek ${ }^{* *}$
}

\begin{abstract}
Concrete deterioration is an important cause of sudden collapse of buildings. Sulfate ion attack is a prominent cause of concrete deterioration. The main cause of sulfate attackinduced damage is ettringite formation in voids, cracks, and contact zones between aggregates and hardened cement paste. Scanning electron microscopy (SEM) images are taken to detect delayed ettringite crystal development in voids. General diffusion equations are utilized to investigate the sulfate ion ingress and the distribution of sulfate ion concentration in concrete related to time. A numerical model demonstrates the relation between the diffusion coefficient and concentration. A computer program is developed to model the problem of ion diffusion in concrete. The sulfate attack on concrete by one-dimensional diffusion process is simulated. The numerical model demonstrates the relation between the diffusion coefficient and concentration.

The computer program is developed to calculate the SO4 ion diffusion. Compared with the SO4 ion diffusion coefficient in free water, the high diffusion value and the low compressive strength of concrete specimen are the values of concrete deterioration. The experimental program included mix design of 6 concrete mixes. The parameters of the experimental program were: cement type and content, presence of silica fume, sulfates concentration, and exposure period.

The results show that, the concrete deterioration by sulfate attack is strongly influenced by the sulfates concentration in the aggressive medium and the exposure time. There is a linear relation between strength loss and concentration for mixes containing silica fume. Ordinary Portland cement (OPC) concrete limits the strength reduction about $10 \%$ after one year exposure to 5,000 ppm sulfate solution. In case of 100,000 ppm sulfate solution, the reduction is $(13.1 \%)$. Increasing the cement content increases the strength loss by sulfates attack even in the presence of silica fume.
\end{abstract}

\footnotetext{
Ph.D. Civil Engineering Branch.
} 
Proceedings of the 6th ICCAE Conf. 16-18 May, 2006

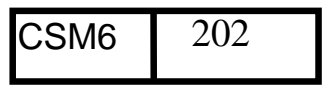

${ }^{* *}$ Lecturer, Ph.D., Civil Engineering Department, Military Technical College. Cairo. Egypt.

Keywords: Dolomite concrete, sulfate ion, compressive strength, computer program, sulfate ion diffusion, Ettringite and Gypsum formation.

\section{INTRODUCTION}

Concrete is a porous material. The porosity is a main phenomenon which is responsible for concrete deterioration. Concrete may also be exposed to cracking due to expansion, shrinkage, and swelling. One of environmental problems arises when the buildings are constructed near fertilizer factories. This paper discusses the concrete deterioration due to sodium sulfate attack. Experimental and analytical studies are developed to avoid concrete buildings sudden collapse due to sulfate attack. The porous system and micro cracks permit for different substances to penetrate into concrete and attack the different elements. The chemical reaction between the substances and the cement paste produces ettringite crystals which deteriorate the concrete.

\section{STRENGTH DETERIORATION}

Strength is a measure of the required stress to push a material beyond its failure point. The concrete strength is a function of the cement hydration process. The compressive strength is attributed to the complex concrete micro-structure [6]. The strength deterioration occurs when the concrete microstructure collapses.

The strength change of M1 (OPC (350 kg/m3) with silica fume) is presented in Fig 1 . The results show that there is no change in concrete strength when subjected to fresh water (0 ppm) during the first 30 days of exposure. From 30 to 365 days of exposure, very slight decline in strength (from 0 to 12\%) was noticed as the result of wet/dry cycles exposure without sulfate effect. The wet/dry cycle is defined that the sample is placed in water for different time period and then is placed in air for one day. However, there is no change in strength of the samples exposed to 5,000 ppm solution during the first 60 days of exposure. From 60 to 180 days there is slight decline in strength because the wet/dry cycles with this low Na2SO4 concentration result in low rate of deterioration. Hence, the slight decline in strength was noticed. From 180 to 365 days there is no change in strength because the rate of reaction is very slow as the result of low Na2SO4 concentration (5,000 ppm). There is a slight gradual decline in strength of 25,000ppm during the first 90 days of exposure $(10 \%$ loss in strength). From 90 to 365 days of exposure, the decline continued but with a milder slope (19.9\% loss in strength). The results show that there is slight decline in concrete strength (6.4\%) when exposed to $100,000 \mathrm{ppm}$ sulfate solutions during the first 30 days of exposure. A decline of concrete strength is attributed to leaching happening after 30 days due to the first wet/dry cycle [2]. From 30 days to 60 days of exposure, practically no change in strength because of the absence of concrete deterioration in this stage as a result of no ettringite formation and the formation of the brucite layer on the outer surface of concrete. This layer is strong and impervious. However, it is formed as an intermediate step in the ettringite and thaumasite formation. It appears and disintegrates in cycles as the sulfates-cement paste reaction progress. From 60 to 90 days of exposure, the results reveal that there is sudden drop in samples strength exposed to 100,000 ppm sulfate solution (From 288 to $258 \mathrm{~kg} / \mathrm{m} 3$ ). The 100,000 ppm of Na2SO4 concentration causes high rate of reaction inside pores hence 
deterioration increase. From 90 to 365 days of exposure, practically, there is a gradual mild decline in the concrete strength (reaches $22.5 \%$ of its control strength). M1, containing silica fume and (OPC), has refined pore structure and capillary pipes. However, the M1 strength has a noticeable strength reduction despite the addition of silica fume to the mix (22.5\% drop after 365 days in 100,000 ppm of Na2SO4). However this strength reduction is smaller than that is observed for mixes without silica fume. This may be attributed to the role of silica fume in minimizing the porosity of M1 mix because of its high fineness. The silica fume pozzolanic reaction reduces the calcium hydroxide content. Hence, the formation of ettringite is controlled in M1 mix. [1].

The strength change of M2 (SRC (350 kg/m3) with silica fume) is depicted in Fig 2. Sulfate resisting cement contains very small amount of C3A , therefore, ettringite formation takes place at a very slow rate. Moreover, the existence of silica fume enhances the pore structure within the cement paste leading to reduce diffusion of harmful ions. From 0 to 30 days of exposure, there is practically no change in strength of M2 exposed to $0 \mathrm{ppm}$ solution. Consequently there is very slight decline in strength. The results show that there is a slight gradual decline in samples strength exposed to 5,000 ppm, 25,000 ppm and 100,000 ppm solutions during the first 90 days of exposure (from 0 to $7.8 \%, 8.5 \%$ and $9.2 \%$ respectively). Then, the strength remains practically constant.

The strength change of M3 (OPC (350 kg/m3) without silica fume) is shown in Fig 3. There is practically no change in strength of M3 exposed to $0 \mathrm{ppm}, 5,000 \mathrm{ppm}$, and 25,000 ppm solutions during the first 30 days of exposure. From 30 days till 180 days of exposure, the results present very slight reduction in strength up to $25,000 \mathrm{ppm}$ concentration. In the interval from 180 days till 365 days, the results show practically constant strength values in solution with sulfate concentrations up to $25,000 \mathrm{ppm}$ (about $10 \%$ strength loss). The results show that there is a slight decline in concrete strength when exposed to solution with 100,000 ppm sulfate during the first 90 days of exposure (from 0 to 8.9\%). This is attributed to the starting of ettringite micro-crystals formation and leaching due to the wet/dry cycles. From 90 till 180 days of exposure, the results reveal more rapid reduction (reaches 19\%). 100,000 ppm of Na2SO4 concentration causes high leaching [3, 4]. Exposure to 100,000 ppm Na2SO4 causes drastic drop in strength after 365 days (30\% of the original strength). The absence of silica fume, (OPC (350 kg/m3)) and relatively high w/c ratio result in cement paste with high permeability. The cycles of gypsum formation, ettringite formation, swelling, expansion and leaching occur in a large number of pores. These cycles have considerable effect on strength loss (Fig 3). The presence of water in the pore system of concrete is essential for the migration of reactant ions up to micro-cracks where ettringite deposition occurs [2].

The strength change of M4 (OPC (450 kg/m3) with silica fume) is depicted in Fig 4. The results show that there is slight gradual decline in concrete strength when exposed to solution with 5,000 ppm, 25,000 ppm and 100,000 ppm sulfate content during the first 30 days of exposure while slight increase of strength is observed for specimens in fresh water. From 30 days till 90 days of exposure, there is slight gradual decline in strength of specimens in all concentrations. The strength decline is proportional to the concentration increase. From 90 days till 180 days of exposure, the results reveal gradual mild reduction in strength with concentrations up to $25,000 \mathrm{ppm}$ and rapid sharp reduction with 100,000 ppm concentration (about 26.8\%). The rapid sharp reduction of samples strength exposed to 100,000 ppm solution is attributed to reaction acceleration between sulfates and cement paste due to the high sulfates concentration. From 180 days till 356 days of exposure, Fig 4 shows the continuity of the mild rate of strength reduction with concentrations up to 25,000 ppm, while 
strength has dropped slightly (about 4\%) with 100,000 ppm solution. This is attributed to the slow sulfate reaction with the remaining calcium hydroxide as the result of solution saturation in pores.

The strength change of M5 (SRC (450 kg/m3) with silica fume) is depicted in Fig 5. M5 compressive strength behavior is practically the same as M4 except for specimens in 100,000 ppm solution. For these specimens, strength reduction started at 60 days instead of 90 days. The reduction in strength loss is caused by the presence of silica fume and the high SRC content. Fig 6 shows the strength change of M6 (OPC (450 kg/m3) without silica fume). The mixes M6 and M3 are approximately the same but M6 has higher strength loss for all concentrations. This peculiar observation may be attributed to the higher cement content in M6.

\section{MICROSTRUCTURAL ANALYSIS OF HARDENED CONCRETE}

The SEM studies are focused on the interfacial zone. Fractured surfaces were examined using secondary electron images which clarify the topography of the interfacial zone and the zones surrounding it. It was implemented also to distinguish gypsum and ettringite crystals in the voids and micro-cracks. SEM shows that ettringite crystals have perfect needle-shape with hexagonal cross-section. These crystals are formed in cracks. These crystals resemble each other and tend to grow together in the same direction. Some of the crystals are twinned and they continue to grow separately. Gypsum crystals and ettringite crystals are easily distinguished. Gypsum crystals have plate form like a mass of successive layers of ice, or fish scales. Meanwhile, ettringite crystals are needle-shaped, as shown in Figure 7.

The SEM image of an M3 specimen after 365 days in 5,000 ppm solution (Figure 7), shows a rare phenomenon. Namely, the appearance of both ettringite crystals and gypsum crystals in the same void (mostly, gypsum forms around pores and cracks rims but ettringite forms inside pores and cracks) is shown in Figure8. SEM image of M6 after 365 days consists of both ettringite crystal and micro-crack, as shown in Figure8. The ettringite crystal expand through the upper crack bank. The ettringite needles are hard and sharp [7]. Figure 8 illustrates the needle-like ettringite crystal rested between the two crack banks. The crystal causes microcracks to increase due to its hardness and expansion [5]. M6 loses 58.8\% of compressive strength after 365 days of exposure to $100,000 \mathrm{ppm}$. M6 recorded the highest strength loss because of the higher ettringite propagation rate.

\section{PREDICTION OF DETERIORATION}

A computer program was developed during the course of this research to model the problem of ion diffusion in concrete cubes. This is done by transforming the diffusion problem into non -dimensional space. The transformed model is solved by using a time-marching technique until reaching a point of time at which local ion concentration levels corresponding to the measured concentration values are reached. Performing a reverse transformation on the model leads to values of effective rate of diffusion. The concrete cubes used in the tests were treated to restrict inflow of the ions by completely insulating five of each cube surfaces with ion-impermeable paint. With only one side exposed to the solution, the ion flow can be reasonably treated as a one-dimensional problem (that is, no lateral flow of the ions). The aim of this model is to calculate an equivalent constant diffusion coefficient in several cases of 
exposure time/solution concentration combinations to study the variation of the diffusion process

with attack parameters. The one-dimensional diffusion of ions through concrete can be modeled using the general diffusion equation [8]. A novel mathematical transformation has been employed to combine time and diffusion coefficient in one abstract variable. Hence, it enabled the solution without the priori knowledge of the value of the diffusion coefficient. This approach required comparison with measured sulfates concentration at different depths inside different

concrete mixes. The experimental program results provided a large set of these data for different concrete mixes exposed to different sulfate solutions for different periods of time. Diffusion coefficients are strongly dependent on the mix properties and the aggressive medium salt content. This makes it impractical to depend on the diffusion coefficient value as a criterion for the evaluation of concrete durability. It is better to rely on continuous monitoring of the sulfates concentration at specified depths inside concrete to detect beginning of diffusion coefficient increase because it means that water begins to replace gypsum and ettringite in pores and micro-cracks, hence the continuity of diffusion coefficient increase means water propagation increase in pores and cracks, hence concrete deterioration continue to reach collapse point at diffusion coefficient approaching 1.065 x 10-9 m2/sec.

\subsection{PROGRAM INPUTS}

The numbers in each line describe the following, respectively: Mix code, cube length(meter), external concentration(ppm), time of exposure(days), four internal concentration values(ppm) at incremental equidistant distances (equal dividing of the whole length of specimen.

M3,0.1,100000,60,1529,1311,1010,901

M3,0.1,100000,90,2836,2428,2053,1332

M3,0.1,100000,180,2916,2531,2166,1614

M3,0.1,100000,365,3154,2600,2238,1989

\subsection{PROGRAM OUTPUTS}

The numbers in each line describe the following, respectively: Mix code, external concentration(ppm), time of exposure(days), diffusion coefficient(m2/sec).

M3,100000,60,1.346e-014

M3,100000,90,1.634e-014

M3,100000,180,8.717e-015

M3,100000,365,4.650e-015

\section{CONCLUSIONS}

There is a linear relation between strength loss and concentration for mixes containing silica fume. The slope of the line increases with time. The strength loss concentration relationship takes a bi-linear trend for mixes without silica fume. The change in slope takes place at sulfates content equals 25,000 ppm. Silica fume reduces the deterioration of concrete by sulfate attack. Addition of $10 \%$ silica fume to $350 \mathrm{~kg} / \mathrm{m} 3$ ordinary Portland cement concrete 
limits the strength reduction after one year exposure to 5,000 ppm sulfates solution to (10\%). In case of $100,000 \mathrm{ppm}$ sulfates solution, the reduction is $(13.1 \%)$ Increasing the cement content increases the strength loss by

Sulfates attack even in the presence of silica fume. The mixes with cement content of $450 \mathrm{~kg} / \mathrm{m} 3$ have shown consistent higher strength loss than the corresponding mixes with $350 \mathrm{~kg} / \mathrm{m} 3$. This may be attributed to the fact that increasing cement content means increasing the porous portion of concrete body facilitating the ingress of sulfates.

Finally, the computer program is also developed to model the ion diffusion in concrete cubes.

\section{REFERENCES}

[1] D. P. Bentz, O. M. Jensen, A. M. Coats, and F. P. Glasser. Influence of silica fume on diffusivity in cement-based materials: I. Experimental and computer modeling studies on cement pastes. Cement and Concrete Research, 30(6):953-962, 2000.

[2] M. Collepardi. Damage by delayed ettringite formation - a holistic approach and new hypothesis. Concrete International, 21(1):69-74, January 1999.

[3] Robert L. Day. Development of performance tests for sulfate attack on cementitious systems. Cement, Concrete, and Aggregates, 22(2):169-176, December 2000.

[4] C. F. Ferraris, J. R. Clifton, P. E. Stutzman, and E. J. Garboczi. Mechanisms of Degradation of Portland Cement-based Systems by Sulfate Attack, published by E \& FN Spon, London, pages 185-192. 1997.

[5] E. J. Garboczi. Stress displacement and expansion cracking around a single spherical aggregate under different expansive conditions. Cement and Concrete Research, 27(4):495500, 1997.

[6] P. Kumar Mehta and Paulo J. M. Monteiro. CONCRETE Microstructure, Properties and Materials. Prentice Hall, third edition, 2001.

[7] Jochen Stark and Katrin Bollmann. Delayed ettringite formation in concrete. Technical Report 23-2, Nordic Concrete Research, 1999.

[8] John C. Tannehill, Dale A. Anderson, and Richard H. Pletcher. Computational Fluid Mechanics and Heat Transfer. Taylor \& Francis, second edition, 1997. ISBN: 1-56032-046-X. 
Proceedings of the 6th ICCAE Conf. 16-18 May, 2006 
Proceedings of the 6th ICCAE Conf. 16-18 May, 2006 